Revue d'histoire de l'Amérique française

PEV RUE D.HISTOIRE DE L'AMÉRIQUE FRANÇAISE

\title{
Les Compagnies du Cap-Breton 1629-1647
}

\section{Robert Le Blant}

Volume 16, numéro 1, juin 1962

URI : https://id.erudit.org/iderudit/302172ar

DOI : https://doi.org/10.7202/302172ar

Aller au sommaire du numéro

Éditeur(s)

Institut d'histoire de l'Amérique française

ISSN

0035-2357 (imprimé)

1492-1383 (numérique)

Découvrir la revue

Citer cet article

Le Blant, R. (1962). Les Compagnies du Cap-Breton 1629-1647. Revue d'histoire de l'Amérique française, 16(1), 81-94. https://doi.org/10.7202/302172ar d'utilisation que vous pouvez consulter en ligne.

https://apropos.erudit.org/fr/usagers/politique-dutilisation/ 


\section{LES COMPAGNIES DU CAP-BRETON}

\section{9-1647}

\section{L'ASSOCIATION AVEC CHARLES DANIEL}

Il est bien connu que le fameux capitaine dieppois Charles Daniel, associé de la Cie de la Nouvelle-France sous le $\mathrm{N}^{\circ} \mathbf{1 1 0}$ de la liste rectifiée par nous, fonda au cours d'un traité particulier passé avec elle, ${ }^{1}$ en septembre 1629 , le fort Sainte-Anne au Cap-Breton avec les dépouilles du lord écossais O'Chiltrie dont il avait enlevé un établissement épisodique au Port-auxBaleines, situé à 25 lieues de là. La redoute française pourvue de 8 canons commanda les passes du Grand-Cibou. ${ }^{2}$ Cet établissement de Sainte-Anne abrita les jésuites Vimont et Vieuxpont signalés par les «Voyages de Champlain » comme revenus du Grand-Cibou sur un bâtiment pêcheur en 1630, alors que 12 Français y étaient morts du scorbut. ${ }^{3}$

L'entreprise subsista cependant et Daniel, associé de moitié avec la Cie, repartit, le 26 avril 1631, pour Sainte-Anne où les directeurs de Paris et de Rouen, c'est-à-dire peut-être ceux de la Cie générale et ceux d'une compagnie secondaire jusqu'à présent mal connue, envoyèrent la même année un navire de Dieppe sous le commandement d'Hubert Anselme qu'une intervention anglaise empêcha de dépasser Miscou. A Sainte-Anne, Daniel trouva une situation tragique: le commandant du fort nommé Gaude avait assassiné son lieutenant nommé Martel, originaire de Dieppe. Charles Daniel remit le commandement

1 Extrait imprimé de la cession de la région du Saint-Laurent par la Cie générale de la Nelle-France à une Cie particulière du 15 novembre 1632, Clairambault 381, fo. 101, à la Bib. Nat., Communication du révérend Père Baudry.

2 G. Félix, Voyage à la Nouvelle-France du capitaine Charles Daniel, de Dieppe (Rouen, 1881) in $4^{\circ}$, Réserve, p. Z 358 (39), idem.

3 Biggar, The Works of Samuel Champlain (Toronto, 1928), VI: 181, $8^{\circ} \mathrm{Pa} 78$ bis à la Bib. Nat. 
à un de ses frères et continua l'entreprise avec la Cie de la Nouvelle-France jusqu'au 16 novembre 1632, date à laquelle les marchandises restées au Cap-Breton furent cédées à la Cie particulière du Canada, concessionnaire du fleuve Saint-Laurent à partir de Miscou. ${ }^{4}$ Daniel céda, de son côté, le 6 janvier 1633, à Nicolas Libert le jeune, associé de la Cie sous le $\mathrm{n}^{\circ}$ 104, demeurant rue des Cinq-Diamants, paroisse Saint-Jacques-de-laBoucherie à Paris, son droit d'associé en la Cie de la NouvelleFrance aux conditions de sa propre acquisition, moyennant 3,000 livres. $^{5}$ De son côté, Lamy, secrétaire de la Cie, céda, le 26 février 1633, l'île du Cap-Breton ${ }^{6}$ à un Parisien, Pierre Desportes qui figure lui aussi sur la liste des Cent-Associés avec le $\mathrm{N}^{\circ} 79$.

\section{LA COMPAGNIE PARISIENNE DU CAP.BRETON}

Fils de Louis Desportes, avocat au parlement de Paris, pourvu d'une charge de secrétaire du roi le 26 octobre 1569 , et d'Anne du Poteau, né vers 1568, comme âgé de 13 ans le 3 mars 1581, date de l'inventaire des biens de son père qu'il partagea avec Louis, son frère cadet, Jeanne et Geneviève, ses deux sœurs, suivant acte passé devant Michel Le Vacher, commissaire au Châtelet, Pierre Desportes rendit hommage, suivant acte passé devant Pierre Rougemont, notaire à Mauregard, le 29 juillet 1596, pour 4 arpents de terre mouvants en plein fief de la seigneurie de Mauregard et se maria, suivant contrat du 13 juin 1599, passé devant Saint-Waast, notaire au Châtelet, avec Geneviève Dupuy, petite-fille de Jacques Dupuy l'aîné, fille de Jean-Baptiste Dupuy et de Geneviève Lécuyer. ${ }^{7}$ Il était déjà, lors de la concession du Cap-Breton, l'associé de Jean Belleteste, marchand de Dieppe, inscrit au $\mathrm{n}^{\circ} 105$ de la liste des Cent-Associés avec qui il fit en 1633 un envoi d'une valeur de 6,200 livres à destination $d u$ fort Sainte-Anne, par un navire ayant pour capitaine Michel Moreu. ${ }^{8}$ La société fut complétée, suivant acte

4 Extrait imprimé déjà cité.

5 Minutier central, minutes de Bonot, LXVII, 262, aux Arch. Nat.

6 Idem, Inventaire après décès de Pierre Desportes, 12 août 1645 , minutes de Richer, LI, 262.

7 Idem.

8 Acte de constitution de la Cie du Cap-Breton du 14 décembre 1633, minutes de Bonot, LXVII, 89. 
du 14 décembre 1633, par le renfort de Charles Daniel, noble homme, capitaine pour le roi en la marine de Ponant et de Nicolas Libert le jeune, marchand mercier qui joua un rôle à l'époque dans la fabrication des chapeaux de castor. ${ }^{9}$ Desportes apporta le privilège renouvelable de la traite au Cap-Breton dont il bénéficiait pour 4 ans à partir du $1^{\text {er }}$ janvier 1634. Le fonds commun de 45,000 livres comprenait 3 navires, La Liane d'environ 80 tonneaux achetée 4,750 livres à Desportes et Belleteste; Le Nicolas de 200 tonneaux acheté 10,206 livres à Libert et Belleteste; La Fortune de 200 tonneaux achetée avec 4 canons 6,200 livres à Daniel. Desportes devait avoir un commis à Dieppe et chaque associé devait payer 220 livres pour le passage et la nourriture de toutes personnes qu'il pouvait envoyer à l'établissement, à concurrence de 8 à 10 par navire. Chacun pouvait vendre sa part ou y associer qui bon lui semblerait sans lui conférer voix délibérative suivant contrat en bonne forme dont il fournirait copie. Dès le 20 décembre, du reste, Belleteste céda le quart de son quart à Daniel et celui-ci céda le quart du sien à noble homme Nicolas de Laistre, bourgeois et ancien échevin de Paris, demeurant rue des Lombards, paroisse Saint-Jacques-de-laBoucherie, ${ }^{10}$ marié avec Jeanne Simon, le 7 octobre $1627 .{ }^{11}$

En exécution de l'acte de constitution, Pierre Desportes et Nicolas Libert donnèrent procuration, le 13 janvier 1634 , à $\mathbf{M}^{\mathrm{e}}$ Jean Le Large, leur facteur, demeurant à Dieppe, pour l'avitaillement du Cap-Breton. ${ }^{12}$ Le fort Sainte-Anne, construit à gauche, en haut d'une côte regardant au nord, dans la baie du GrandCibou, large de deux lieues à l'entrée, mais se rétrécissant pendant six à sept lieues, abritait alors plusieurs Pères jésuites qui continuaient d'y résider en éprouvant de la difficulté à convertir les sauvages. ${ }^{13}$ Pierre Desportes se préoccupa dès cette époque

$94^{\circ} \mathrm{Fm}$ 19503, à la Bib. Nat.

10 Addendum à l'acte de constitution déjà cité.

11 Minutes de Cousinet, LI, 482.

12 Addendum à la suite de l'acte de constitution déjà cité.

13 \& Relation de quelques particularités du lieu et des habitants de l'île du Cap-Breton envoyée par le père Julien Perraụlt de la Cie de Jésus, l'an 1634», Jesuits Relations, VIII, 1634-1636: 156, Lk12 1464, à la Bib. Nat. 
d'organiser personnellement des entreprises de culture au CapBreton. Suivant contrat du 6 avril 1634, passé devant Nicolas Herbin, notaire au Châtelet, ${ }^{14}$ il autorisa Mathurin Gauguier, âgé de 34 ans, natif de Maisonneuve, paroisse de Cherves, à une lieue de Mirebeau en Anjou ${ }^{15}$ et la femme de ce dernier, Marguerite Petit, native de Paris, âgée de 28 ans, à construire à leurs frais une maison dans l'habitation du fort Sainte-Anne, leur concédant outre cent arpents de terre, bois et prés à prendre à l'endroit de leur choix dans l'île du Cap-Breton, moyennant la redevance de la trentième gerbe de blé que produiraient les terres, la moitié des terres qu'ils défricheraient en récompense d'une indemnité de 300 livres par an, pendant trois ans, et de leur nourriture assurée pendant la traversée qualifiée voyage qui devait s'effectuer de Dieppe, en février 1635.

L'activité de certains associés comprit le négoce de fourrures qui n'avaient pas été acquises en vertu de leur privilège, car Daniel qui se rendit en Nouvelle-France, en 1634, y passa avec Charles de Turgis, dit de Saint-Etienne-de-La-Tour, et Guillaume Desjardins, sieur de Saint-Val, un marché pour 800 peaux de castors et orignaux ${ }^{16}$ livrables au Cap-Breton au mois d'août 1635. Desportes, Libert et Claude de Laistre, procureur de son père, Nicolas, donnèrent, le 29 mars 1635, une procuration à Philippe Le Roy pour réclamer l'acte à Charles Le Roy, capitaine de la marine à Dieppe. ${ }^{17}$ Ils en donnèrent une autre, à la même époque, pour diriger le transport des victuailles et marchandises au Cap-Breton à Charles Daniel,18 et noble homme Claude de Laistre devint le gendre de Desportes en épousant sa fille Marie, suivant contrat passé devant Nicolas Herbin, le 15 juillet $1635 .{ }^{19}$ Cette année 1635 fut celle du dernier exercice de la Cie, car Desportes s'associa au début de 1636 avec le marchand rochelais

14 LVIII, 123, aux Arch. Nat.

15 Actuellement Cherves, canton de Mirebeau, arr. de Poitiers.

16 Procuration du 29 mars 1635, Bonot, LXVII, 94.

17 Idem.

18 Idem, addendum.

19 LXVIII, 124. 
Jean Tuffet qui figure au $\mathrm{N}^{\circ} 38$ de la liste des Cent-Associés;20 mais les affaires avec Charles de Turgis n'étaient pas terminées. Tandis que Charles Daniel avait cédé, suivant acte du 24 février 1638, pour 600 livres, à Nicolas Libert, sa créance contre la Cie de la Nouvelle-France pour l'habitation de Sainte-Anne, ${ }^{21} 400$ peaux de castors, loutres et orignaux restant à livrer sur le marché avec La Tour porté à 1,200 peaux, firent l'objet d'un accord du 11 janvier 1639 entre Daniel, Libert et Guillaume Desjardins, agissant, tant pour lui-même, que comme intendant de Charles de Turgis. ${ }^{22}$ Celui-ci se trouvait en difficulté pour livrer le solde ou en avait trouvé un prix plus avantageux, car il accepta de payer pour la résiliation, une indemnité de 4,800 livres lui paraissant élevée. Les anciens associés de la Cie du Cap-Breton avaient convenu d'une répartition d'apports particulière, Desportes et Libert étant intervenus chacun pour cinq seizièmes, Daniel pour quatre et Nicolas de Laistre pour deux. Libert se trouva done créancier pour 1,500 livres, et Daniel pour 1,200. Sur ces 2,700 livres, Desjardins en paya comptant 1,687 livres 10 sols, le solde de 1,012 livres devant être réglé à la fin du mois d'août. Nicolas de Laistre accepta également pour sa part de 600 livres, un versement dans les mêmes conditions. Desportes suivit le mouvement le dernier janvier. ${ }^{23}$

\section{LA CIE DESPORTES-JEAN TUFFET}

Rochelais catholique réfugié à Bordeaux, ${ }^{24}$ Jean Tuffet avait été un des directeurs de la Cie de la Nouvelle-France pendant la période tragique de 1628 à 1631 avant de regagner son domicile. ${ }^{25}$ Il engagea du personnel à destination du Cap-Breton,

20 Transaction entre Desportes et André Tuffet du 30 décembre 1642, registre de Teuleron de 1642 , fo. 165 , vo., aux Archives de la CharenteMaritime et de M. Delafosse, Revue d'Histoire de l'Amérique française, IV (mars 1951) : 474-5, $8^{\circ} \mathrm{Pa} 535$, à la Bib. Nat.

21 Minutes de Camuzet, XXXV, 151.

22 Idem, 157.

23 Minutes de Bonot, LXVII, 102.

24 Acte passé devant Cherbonnier, notaire à Bordeaux, le 4 décembre 1648, E 3206, aux Arch. Dép. de la Gironde.

25 En tenant compte de la rectification ci-dessus, notre article: « La Cie de la Nouvelle-France et la restitution de l'Acadie $\gg$, Extrait de la Revue d'Histoire des Colonies, $80,8^{\circ} \mathrm{Lc12} 293$, et $8^{\circ} \mathrm{Pa}$, pièce 4 , à la Bib. Nationale. 
le 5 avril $1636,{ }^{26}$ et traita, le 10 avril suivant, avec Jean Davanon, sieur de Saint-Germain qui souscrivit un engagement pour commander les habitants du Cap-Breton en qualité de capitaine et gouverneur avec la responsabilité des magasins et des distributions de vivres. ${ }^{27}$ Natif de Libourne, ce dernier consentit une procuration en faveur de son beau-frère, Florent Parguier, maître-chirurgien à Libourne, et reçut sa commission de Desportes, promettant d'agir pour le service du roi, sous l'autorité de la Cie de la Nouvelle-France. ${ }^{28}$

Tuffet engagea, le $1^{\text {er }}$ mars 1637 , un tailleur d'habits et un chirurgien nommé Jean Mulot pour l'habitation du Fort SteAnne au Cap-Breton, ${ }^{29}$ puis passa, comme procureur de Desportes, le 2 avril suivant, une charte-partie avec Geraud de Punault, maître du navire La Marie, de Saint-Jean-de-Luz, aux fins de livrer au fort Sainte-Anne 32 tonneaux ou environ de marchandises à Jean Davanon, capitaine et gouverneur de l'île du Cap-Breton. ${ }^{30}$ Il engagea, entr'autres un nommé Chauson et sa femme, le 28 mars $1638^{31}$ puis fréta, le lendemain, le navire Le Soleil, de 140 tonneaux, à Laurent Cadou, marchand du bourg de Saint-Gilles en Poitou, qui en était co-propriétaire, pour, par l'intermédiaire de Martin du Halde, marinier de Biarritz, maître de ce navire, faire la pêche au Cap-Breton, y transporter vivres et marchandises, puis recevoir les pelleteries de Davanon. ${ }^{32}$. Le 15 janvier 1639, Tuffet, agissant encore pour lui-même et Desportes, acheta à honorables hommes Louis Allaire et Jean Caron, marchands de La Rochelle, la moitié du navire Le Saint-Pierre de 130 tonneaux ou environ avec les effets, munitions et marchandises en partie restés au CapBreton qu'ils avaient chargés en société dont Allaire avait les

26 Registre de Teuleron, 1636, fos. 23, vo. 24, 26, aux Arch. de la Charente-Maritime.

27 Idem, fo. 28.

28 Idem.

29 Idem, Liasse de Teuleron de 1637, à la date.

30 Registre de Teuleron de 1637, fo. 32 , vo.

31 G. Debien, «Les engagés pour le Canada au XVIIe siècle 》, Revue d'Histoire de l'Amérique française, op. cit., VI: 221, Registre de Teuleron de 1638, à la date.

32 Idem, fo. 24. 
deux tiers et demi, Caron un seizième. ${ }^{33}$ C'est dans un acte du 14 mars 1640 qu'il fut pour la première fois question du fort Saint-Pierre du Cap-Breton. Tuffet engagea, toujours comme associé de Desportes, Denis Bellotoire, capitaine de marine, demeurant au château de l'île d'Oléron pour y partir sur un navire commandé par du Halde, y exercer pendant trois ans, aux appointements annuels de 200 livres, la charge de lieutenant sous Davanon de Saint-Germain et, en cas de retour en France de ce dernier, suivre les ordres de Louis Parguier, commandant pour Desportes au fort Saint-Pierre. ${ }^{34}$

Jean Tuffet recruta des engagés, dont Laurent de Huisseau, chirurgien, natif d'Orléans, le 29 avril $1640^{35}$ et passa une charte-partie, le 13 mai suivant, avec Martin du Halde pour pêcher la morue et décharger des marchandises au Cap-Breton..$^{36}$ Indisposé lorsque son fils André, substitut du procureur général des salines de La Rochelle, réalisa pour lui, le $1^{\text {er }}$ avril 1641, les engagements de Jean Bonnard, maître cloutier et de sa femme, puis d'André Goisin, charpentier, pour servir son fils Louis, commandant le fort Saint-Pierre, ${ }^{37}$ il passa encore, pour son compte et celui de Desportes, avec Martin du Halde, pour le navire Le Saint-Pierre, à destination du Cap-Breton, une chartepartie du 3 avril 1641, revêtue de sa signature. ${ }^{38}$

Jean Tuffet mourut avant le 22 mars 1642, laissant des affaires dont la mauvaise situation avait des causes plus amples que la carence de Desportes qui, depuis 1639, avait cessé de fournir les subsides correspondant à sa part. ${ }^{39}$ Les biens de Tuffet furent saisis par de nombreux créanciers, dont Samuel Gallet, sieur de Thirac, pour 8,600 livres, Joachine Gombauld, sa veuve, fit opposition à la saisie comme créancière de 10,000

33 Acte du 24121639 , reg. de Teuleron 1639-40, fo. I.

34 Idem, à la date.

35 Idem.

36 Idem.

37 Reg. de Teuleron de 1641, à la date.

38 Idem, fo. 48 , vo., G. Debien, «Les engagés pour les Antilles》, Revue d'Histoire des Colonies (1951) : 81, op. cit. déjà citée.

39 Transaction entre Desportes et André Tuffet du 30 décembre 1642, 
livres représentant ses deniers dotaux depuis 1605; Etienne $\mathrm{I}^{\text {er }}$ Tuffet, frère du défunt, fit de même pour 617 livres. $\mathrm{M}^{\mathrm{e}}$ Jean Fayet, ancien avocat, curé de Saint-Aubin, paroisse SainteEulalie, à Bordeaux, son gendre, veuf de sa fille Marie Tuffet qui avait laissé des enfants, ${ }^{40}$ abandonna la succession. Fayet remit à son beau-père André pour la transporter à Gallet la part de 21 lieues de marais salants indivis avec Etienne ${ }^{\text {er }}$ Tuffet au petit Transon, dans l'île d'Oléron, reçue pour Marie Tuffet en équivalence d'une dot de 10,000 livres. André Tuffet n'avait accepté la succession de son père que sous bénéfice d'inventaire. Il vendit à honorable homme Ogier Duchanin, pour 8,000 livres destinées à sa mère la moitié d'une grande maison de La Rochelle, rue du Perrot et au pont de Verdon ${ }^{41}$ qui avait été saisie avec un petit héritage à Ronsay. Comme Joachine Gombault était débitrice d'Hélie Mocquet, autre marchand rochelais, son gendre réclama pour lui rendre service la dot de 10,000 livres et se contenta finalement de 12 lieues de marais salants au Tublat, à Santron.

\section{LES COMPAGNIES ROCHELAISES D'ANDRE TUFFET}

Avocat à la Cour du parlement de Paris, demeurant à La Rochelle, frère de Louis Tuffet, neveu de noble homme Etienne $1^{\text {er }}$ Tuffet, avocat au parlement et au siège présidial de La Rochelle, marié avec Marie Dubois, il était cousin germain des deux fils de ces derniers: Jacques et Etienne II Tuffet, avocat comme son père au présidial de La Rochelle. Jacques Tuffet épousa, suivant contrat du dernier janvier 1641 et les rites de la religion catholique, Catherine Gaigneur, fille de feu honorable homme $\mathrm{M}^{\mathrm{e}}$ Jean Gaigneur, procureur au siège présidial de La Rochelle et de Madeleine Mondot. Etienne II Tuffet, juge civil et criminel au même siège, épousa, suivant contrat du même jour, Madeleine Gaigneur, sœur de Catherine. ${ }^{42}$ André Tuffet

\footnotetext{
40 Procuration du $1^{\text {er }}$ janvier 1648, E 3206 à la date, aux Archives de la Gironde.

41 Actes du 4 décembre 1648 passé devant Cherbonnier, notaire à Bordeaux, idem, et du 3 juillet 1655, Teuleron nouveau, 1648-1649, aux Arch. Dép. de la Charente-Maritime.

42 Registre de Teuleron de 1641, fos. 12 et 13, vo., Idem.
} 
agissait pour le compte de Pierre Desportes, le 22 mars 1642, lorsqu'il affréta le navire Le Saint-Pierre, commandé par de Combes. Cette opération effectuée avec le concours des Rochelais Louis Gaigneur et Jacques Papin ${ }^{43}$ avait pour but d'aller faire la pêche de la morue au Cap-Breton et de faire remettre des marchandises en échange de pelleteries à Louis Gaigneur, commandant au fort Saint-Pierre que de Combes s'engageait à aviser de son arrivée. ${ }^{44}$

Le capitaine du Saint-Pierre arrêta, le 8 juillet au CapBreton celui de la frégate hollandaise, Le Jupiter, nommé Jeune Dricq, âgé de 26 ans. La prise fut conduite à La Rochelle, puis à Brouage où son adjudication fut demandée au substitut du procureur du roi de l'Amirauté de Brouage par Louis Gaigneur, sieur de Croix-Chapeau, capitaine des habitations Sainte-Anne et Saint-Pierre au Cap-Breton. L'audition du capitaine Dricq resté malade à bord du Saint-Pierre fut demandée le 23 octobre au juge de La Rochelle de Mirande par André Tuffet agissant encore tant pour lui que pour Pierre Desportes. ${ }^{45}$ Le Hollandais reconnut qu'il avait fait le troc avec les sauvages. Sur ces entrefaites, Pierre Desportes délégua pour traiter avec André Tuffet l'avocat Antoine Cheffault, alors un des directeurs de la Cie des Cent-Associés sur la liste desquels on le trouve sous le $\mathrm{n}^{\circ} 62$. Tuffet prit l'engagement, le 30 décembre 1642 , de rendre compte, tant des opérations de Jean Tuffet que des siennes, étant donné qu'après le décès de son père, il avait, tout en faisant sommer Desportes de faire connaître ses intentions, pris sur lui d'envoyer Le Saint-Pierre pour secourir les hivernants. Cheffault ayant admis que le compte de Tuffet était créditeur, il fut convenu que ce dernier conserverait le produit des «retours» de 1643 avec celui de la confiscation portant notamment sur les pelleteries échangées par le capitaine Dricq. Tuffet prit la ferme du Cap-Breton pour 3 ans, soit jusqu'au 1er janvier 1646 avec le droit de confisquer les bâtiments dont l'équipage y viendrait

\footnotetext{
43 Sur cette famille, Acte de partage dans registre de Juppin de 1637 , fo. 55 , Idem.

44 Registre de Teuleron de 1642, fo. 34, idem.

45 B 5656, idem, La mention de l'année a disparu du document par suite de l'usure.
} 
traiter, renonçant aux appointements réclamés par son père, moyennant l'abandon à son profit des vivres et des mâtereaux se trouvant sur place, le droit de se servir des canons, des autres armes et du matériel de ménage devant être restitués à la fin des opérations. ${ }^{46}$

Les difficultés financières personnelles d'André Tuffet n'étaient pas terminées. Qualifié noble homme et avocat, se portant fort pour son frère Louis, il convint, le 9 mars 1643, avec son oncle Etienne 1er, de conserver comme commune une maison aux planchers salis, Grande-Rue à La Rochelle, occupée comme locataire par Jean Pinard, susceptible d'exiger le curage des privés dont les immondices coulaient dans la cour, ${ }^{47}$ mais il vendit, le même jour à Ogier, alias Auger Duchanin, deux autres maisons situées aussi Grande-Rue. ${ }^{48}$ Cependant, le Cap-Breton était passé entièrement entre les mains des Rochelais. André Tuffet recruta des engagés les 11 février et 2 mars $1643^{49}$ et affréta, le 21 mars suivant avec Louis Gaigneur et Jacques Papin, Le Saint-Pierre, commandé, cette fois, par Jean Bouchet, pour aller faire la pêche aux côtes du Cap-Breton sous la direction de Louis Gaigneur et de Louis Tuffet, commandant l'île. ${ }^{50}$ La composition des Compagnies du Cap-Breton était décidément éphémère, car André Tuffet était associé, le 16 janvier 1644, avec Dominique de Chevery et Ogier, alias Auger Duchanin, marchands de La Rochelle. Ce dernier devait s'occuper longtemps des affaires de la Nouvelle-France, car on le retrouvera, le 14 janvier 1653, commissaire de la majorité des créanciers de la communauté de Québec. ${ }^{51}$ Les nouveaux associés recrutèrent, alors de nombreux engagés pour Le Saint-Pierre, du 8 janvier au 30 mars $1644^{52}$ et, comme bourgeois du Petit-Saint-

\footnotetext{
46 Registre de Teuleron de 1642 , fo. 165 , vo., signalé par M. Delafosse, Revue d'Histoire de l'Amérique française, IV (mars 1951) : 474-5, $8^{\circ} \mathrm{Pa} 535$, à la Bib. Nat.

47 Registre de T'euleron de 1643 , fo. 21 , vo.

48 Idem, fo. 28.

49 G. Debien, «Les Engagés pour le Canada », op. cit., 227.

50 Registre de Teuleron, 1643 , fo. 32 , vo.

51 Liasse de Teuleron, de $1653-1658$.

52 Debien, «Les Engagés pour le Canada», op. cit., 374, registre de Teuleron, 1644, fo. 165 , vo.
} 
Pierre, commandé par Pierre Bertrand, le 30 mars 1644, Adam Delcaourdy, marchand de Saint-Jean-de-Luz, pour faire le troc avec les sauvages suivant les ordres de Louis Tuffet, Joanis Delcaourdy, fils d'Adam, et Laurent de Harribillague, ainsi dénommé d'après sa signature, qui devaient, comme mariniers, obéir aussi à Louis Tuffet. ${ }^{53}$

Les changements étaient loin d'être terminés, car, suivant acte passé le 4 février 1645 en la maison de Cheffault, rue Simon-le-Franc, à Paris, Pierre Desportes céda une grande partie des droits qu'il tenait de la Cie de la Nouvelle-France au CapBreton, à partir du 1er janvier 1646, à Gilles Guignard, sieur de La Fontaine, garde ordinaire provincial de l'Artillerie de France, département et gouvernement de Poitou, La Rochelle, pays d'Aunis, Brouage, Saintonge et Angoumois, demeurant à La Rochelle. L'abandon comprenait, moyennant une rente annuelle de 300 livres rachetable pour 5,400, l'exercice de la traite dans toute l'île, pour une valeur d'estimation à fixer, payable en France, en 1646 avec un profit de 12 1/2 pour cent, tous les meubles, ustensiles et hardes appartenant au cédant qui se trouvaient au Cap-Breton; enfin, à charge de rendre hommage à ce dernier, ou à ses hoirs, avec prestation, dans le délai d'un an après chaque mutation, de deux robes de castor de cinq à six (sic) et de deux peaux d'orignaux, le fort Sainte-Anne avec un territoire de terres défrichées, prés, bois, lacs, rivières et autres lieux, délimité par une ligne droite aboutissant, en traversant l'île à Soudy et par la mer de tous les autres côtés. ${ }^{54}$ A l'exception de la traite, Desportes se réservait le reste de l'île pour faire défricher, envoyer des laboureurs et nourrir des bestiaux. Cette entreprise comprenait l'habitation de SaintPierre située au sud sans le cap de Panouers. Guignard était constitué comme procureur pour retirer les meubles de Desportes des mains des habitants de Saint-Pierre. Tuffet, Duchanin et de Chevery passèrent la main pour le commerce du CapBreton à l'acquéreur ${ }^{55}$ qui s'intitula, vraisemblablement en vertu

53 Idem, folios 221, vo. et 222.

54 Minutes de Cousinet, LI, 514, aux Archives Nationales.

55 Registre de Teuleron de 1645-6, fos. 55 et 56. 
d'une autre cession que nous n'avons pas retrouvée, sieur des forts Sainte-Anne et Saint-Pierre au Cap-Breton. ${ }^{56}$ Il fut décidé que Guignard ramènerait le personnel qui s'y trouvait. Etant donnés les grands frais entraînés par un tel voyage et la nécessité d'envoyer chaque année un navire au Cap-Breton, les consorts Tuffet, par acte du 5 mai 1645, autorisèrent Guignard à passer avec son navire Les Étoiles dans l'île où Louis Tuffet, après l'avoir mis en possession, devait procéder avec lui à l'inventaire de toutes les marchandises de traite, ustensiles, barques, chaloupes et autre matériel. ${ }^{57}$

Louis Tuffet confia cependant, pour le compte de la Cie qui l'employait à Paul Bertrand, maître du navire de Guignard, 200 peaux d'orignaux et 1 barrique de castors marqués en conformité d'un connaissement que Bertrand et son équipage refusèrent de décharger sous prétexte qu'ils n'étaient pas réglés par Guignard. Sur la requête de Duchanin agissant pour lui et ses associés, le juge de l'Amirauté de La Rochelle, de Mirande, sieur de Fragnié se transporta sur le bâtiment. Bertrand demanda des précisions sur le paiement de son fret et fit état d'une saisie portant sur ce qui appartenait à Tuffet diligentée par Pierre Rostan, marchand de La Rochelle. Le magistrat ordonna que sans préjudicier aux droits des parties, les pelleteries seraient portées en la maison de la veuve Bouchet, chargée de les garder jusqu'à nouvelle décision. ${ }^{58} \mathrm{Au}$ cours de cette même année 1645, une pinasse dépendant de l'habitation de Tuffet au Cap-Breton fut saisie par d'Aulnay. Un Récollet qui se trouvait à bord obtint heureusement la libération de l'équipage, ${ }^{59}$ mais le 4 septembre 1647 , sommation fut faite à Jacques Maillet et René Guignard, commandant au fort SaintPierre, d'avoir à le remettre à Charles de Menou dans le gouvernement duquel il se trouvait compris. ${ }^{60}$ Il s'agissait évidem-

56 B 192, fo. 44, aux Arch. Dép. de la Charente-Maritime.

57 Registre de Teuleron, 1645-6, déjà cité.

58 B 5656, aux Archives de la Charente-Maritime.

59 Déposition de Pierre Sauvic, du 8 novembre 1645, Provisoire 77, aux Arch. Départementales de la Charente-Maritime.

60 Colonies C II D I, fo. 81, aux Arch. Nationales. 
ment de l'application des lettres patentes du mois de février 1647 qui ne tinrent pas compte des droits de la Cie de la Nouvelle-France. Elles rendirent d'Aulnay tout-puissant en Acadie, mais leur révocation entérina la ruine de sa famille.

André Tuffet disparut du Cap-Breton et quitta même La Rochelle entre le 4 décembre $1648^{61}$ et le 7 novembre 1664, date à laquelle, toujours avocat au parlement de Paris et intitulé noble homme, demeurant rue Regratière, paroisse Saint-Louis, en l'île Notre-Dame, il intervint dans les effets d'un arrêt du parlement de Paris rendu le 26 août 1633 en la chambre de l'Edit. ${ }^{62}$ Il n'avait pas changé d'adresse, le 23 septembre 1671, lorsqu'héritier et exécuteur testamentaire de Françoise Jubault de Beauregard, femme de Me Gilles Poupardin, conseiller en l'élection de Bourges, il passa avec ce dernier, remarié alors avec Marie Cordonnoy, ${ }^{63}$ une transaction rappelant le contrat de mariage de Françoise Jubault, passé le 6 juin 1635, devant Roussel, notaire au Châtelet. ${ }^{64}$ Pierre Desportes mourut entre le 2 août 1645, date d'un codicile en faveur de sa fille Louise ${ }^{65}$ et le 12 août suivant, date à laquelle sa veuve fit inventorier ses biens. ${ }^{66}$ Sa succession ne fut acceptée que sous bénéfice d'inventaire, et de son mariage restaient au moins 5 enfants, Pierre II, Arnould, Louise, Marie et Anne.

Pierre II Desportes, sieur de Choisy demeurait en sa maison de Lignières, au village de ce nom, ${ }^{67}$ lorsqu'il en gratifia, le 26 novembre 1656, sa sœur Anne et François Bonnefoy, tuteur de Claude et Marguerite de Marle, ses neveu et nièce. ${ }^{68}$ Arnould, écuyer, sieur du Godeau, fut avocat au parlement de Paris. Louise épousa Charles de Marle, conseiller du roi, rece-

\footnotetext{
${ }^{61}$ Minutes de Teuleron nouveau, 1648-59, acte du 3 juillet 1655, aux Archives de la Charente-Maritime.

62 Minutes de Teuleron, 1664-7, Idem.

63 y 223, fo. 51, vo., d'après les minutes de Jean François, LXXXIV, 180, aux Archives Nationales.

64 Acte perdu ne figurant pas dans LXXXI, 9.

65 y 185 , fo. 18.

66 Minutes de Richer, LI, 262, idem.

67 Soisy-sur-Seine.

68 y 193 , fo. 347 , vo., d'après minutes de Richer, LI.
} 
veur des Tailles en Picardie, et mourut en 1645. Lors du contrat de mariage entre Marie Desportes et Claude de Laistre, celui-ci fut assisté par ses parents, maîtres Guillaume et Nicolas II de Laistre, ses frères, par Marie Boué, veuve d'honorable homme Nicolas Simon l'aîné, marchand bourgeois de Paris, sa tante maternelle, par honorable homme Denis Poisson, bourgeois de Paris, son oncle. La fiancée qui eut une dot de 50,000 livres, fut assistée par Pierre Parfaict, conseiller en la Grande Chancellerie, Jacques Boucheron, correcteur en la Chambre des Comptes, Claude Boucheron, écuyer, sieur de Tillières, Charles Le Boulanger, ses oncles, par $\mathrm{Me}$ Arnould Lallemant et Jacques Broussel, conseiller au parlement, ses cousins. Anne Desportes épousa, suivant contrat du 5 janvier 1641, passé devant Richer et Bonot, notaires au Châtelet, ${ }^{69}$ messire René Frotté, conseiller du roi, trésorier général de France en la généralité de Bordeaux, fils de défunt messire Honoré-Pierre Frotté, conseiller du roi, grenetier et sénéchal d'Ingrande en Anjou et de demoiselle Anne Corneillau. La fiancée fut assistée entre autres, par demoiselle Marguerite Danes, veuve de Me N. Le Prebstre, conseiller au parlement de Paris (c'est-à-dire Claude Le Prestre), sa cousine, s'agissant des parents de Denis et Louis Le Prestre, associés de Notre-Dame de Montréal, $^{70}$ par Me Pierre Broussel, conseiller au parlement, commissaire aux requêtes, son cousin, par noble homme Louis Le Noir, trésorier général des Fermes, par Me Charles Le Noir, conseiller en la Cour des Aides, par Laurent Le Noir, avocat au parlement, par noble homme Antoine Le Comte, conseiller du roi, contrôleur général des guerres son cousin. Il convient de penser qu'elle hérita du Cap-Breton et que René Frotté représente le sieur Frotté qui en demanda 2,000 livres à Nicolas Denys. ${ }^{71}$

\section{ROBERT LE BLANT, 24 rue de Varize, Paris $X V I^{e}$}

69 Carrés d'Hozier 278, art. Frotté, d'après minutes de Bonot, LXVII. 70 Minutes de François Fournier, donation du 2 mai 1640, XCIX, 157. 71 Lettres de Nicolas Denys des 27 juin et 5 juillet 1657, Fonds La Bourdonnaye-Montluc, $2 \mathrm{E}$ suppl. 248, aux Archives départementales d'Illeet-Vilaine. 